\title{
Metodología para la reducción de tiempos en la adquisición de suministro en empresas del sector eléctrico.
}

\author{
DOI: https://doi.org/10.17981/bilo.3.1.2021.02 \\ Fecha de Recepción: 01/04/2021. Fecha de Publicación: 05/05/2021 \\ B Rodríguez -Viloria \\ Brodrigu25@cuc.edu.co \\ Grisales- Peñaloza D. \\ Dgrisale1 @ cuc.edu.co \\ Almanza -Acendra, L \\ Lalmanza@cuc.edu.co \\ Medina -de León, $P$. \\ Pmedina1@cuc.edu.co \\ Barrera -Maldonado, $\mathrm{S}$ \\ Sbarrera@cuc.edu.co \\ Alexander Troncoso-Palacio \\ atroncos1@cuc.edu.co \\ Departamento de Productividad e Innovación \\ Universidad de la Costa. Barranquilla, (Colombia)
}

Resumen. En el presente artículo, se usaron algunas herramientas de calidad para identificar dificultades presentadas en el área de suministros de una empresa constructora en el sector eléctrico, se tomaron como referencias artículos y revistas científicas relacionadas al suministro en el área eléctrica y sobre todo que aspectos relevantes se han tenido en cuenta para llevar la investigación a cabo. En primera instancia se elaboró una entrevista a algunos trabajadores de empresas de este sector donde se seleccionaron algunas de las causa que ocasionan el problema, como segundo paso se utilizó el diagrama de Ishikawa en el cual se identificaron las posibles causas y fenómenos que causaron el problema en el área de suministros, Después se utilizó el diagrama de Pareto con las causas identificadas en las anteriores herramientas, así se identificó los pocos vitales que representan el $80 \%$ de la frecuencia, ya con los pocos vitales identificados se emplea una tabla de frecuencia para llegar a la causa principal del problema en el área de suministros, en el cual la causa identificada fue exceso de pasos en los procesos y así se concluyó con la recomendación de la metodología a seguir para la reducción de tiempo en la adquisición de suministro.

(C) The author; licensee Universidad de la Costa - CUC.

BILO vol. 3. no. 1 Enero - Junio, 2021

Barranquilla. ISSN Online 2711-3280. 


\section{Introducción}

La cadena de suministro de la industria constructora en el sector eléctrico [1,2] ha sido objeto de innumerables estudios cuyos resultados se han aplicado a distintos sectores de esta, entre ellos el sector eléctrico, siendo influenciado negativamente, disminuyendo la producción por la pandemia del Covid-19 [3, 4] , y no solo ella, sino todo el mercado global.

Se quiere identificar las principales dificultades que presenta la empresa en el sector eléctrico en el aprovisionamiento de suministro [5], dicho proyecto investigativo tiene como objetivo analizar e identificar las causales inherentes en la problemática, por medio de la utilización de las herramientas de calidad [6, 7, 8] entre ellas el Diagrama Ishikawa, Diagrama de Pareto [9], Flujograma, hallando la raíz y obteniendo solución óptima ante la problemática presente.

\section{Revisión de la literatura}

La desviación en la adquisición de suministro puede alterar todo la cadena de una manera significativa, la cadena de suministro $[10,11,12]$ debe ser tratada como un sistema completo, lo que quiere decir que cualquier cambio altera el resultado de esta. La cadena de suministro hace parte del conjunto de elementos que permiten que las empresas cuenten con la organización necesaria para llevar a cabo el desarrollo de un producto o servicio y que este cumpla el objetivo principal que es satisfacer las necesidades del cliente final. El control de la calidad se aplica a todos o la mayoría de los procesos de las empresas $[13,14,15]$, con la finalidad de dar soluciones a los problemas que no permiten a la empresa avanzar.

El concepto de calidad radica desde tiempos ancestrales, actualmente la calidad hace parte no solo de nuestro entorno empresarial sino personal, hoy en día se ve reflejado la implementación de herramientas en las industrias, algunas investigaciones como [16] hace referencia a la ejercitación exhaustivas incorporadas en controles de calidad desde la materia prima hasta el producto terminado, por medio de una lista de chequeo se alcanzó los objetivos de esa investigación. En el artículo [17] se redujo el porcentaje de demanda insatisfecha incrementando la productividad, identificando como causas principales la inexistencia de un método de trabajo establecido, presencia de mermas en el proceso, tiempo de producción elevado y distribución de planta ineficiente, logrando encontrar propuesta de mejora del proceso de producción. Las investigaciones explican la finalidad de proponer mejoras en los procesos críticos, identificando las causas que generan la demora, utilizando el diagrama de Ishikawa [18] y mediante el diagrama de Pareto. También [19, 20] afirma que se puede implementar el diagrama de flujo como referencia de la secuencia de actividades que se están realizando en una operación e identificar los fallos en el proceso.

\section{Metodología}

Para esta investigación, se utilizó una metodología mixta en la cual se recolecto, analizó y combinó datos cuantitativos y cualitativos en el caso estudio en el área de suministros en una empresa del sector eléctrico, se decidió estudiar las causas por las cuales se originaba pérdida de tiempo en la adquisición de suministros de la empresa, por el cual primeramente se recopiló información a través de una entrevista con los colaboradores para identificar cuál es la causa que más se presenta en la empresa que puede estar ocasionando la perdida de tiempos en la adquisición de suministro, para el cual luego se realizó un diagrama de Ishikawa en donde se observaran causas y efectos de la problemática presentada, como se muestra en la figura 1. Más tarde ya con los datos cuantificados se procedió a usar el diagrama de Pareto para identificar las causas más potenciales que inciden en el problema de la empresa para finalmente dar las respectivas sugerencias según las posibles soluciones que se puedan determinar para mejorar los procesos en la empresa en este sector.

(C) The author; licensee Universidad de la Costa - CUC.

BILO vol. 3. no. 1 Enero - Junio, 2021

Barranquilla. ISSN Online 2711-3280. 


\section{Desarrollo}

En primera instancia, se utilizó herramienta para recopilar información, referente a las posibles causas que están relacionadas con la problemática presente, aparte de ello se construyó la herramienta $6 \mathrm{M}$ [21] para organizar las causales de dicho diagrama y así tener una percepción más clara.

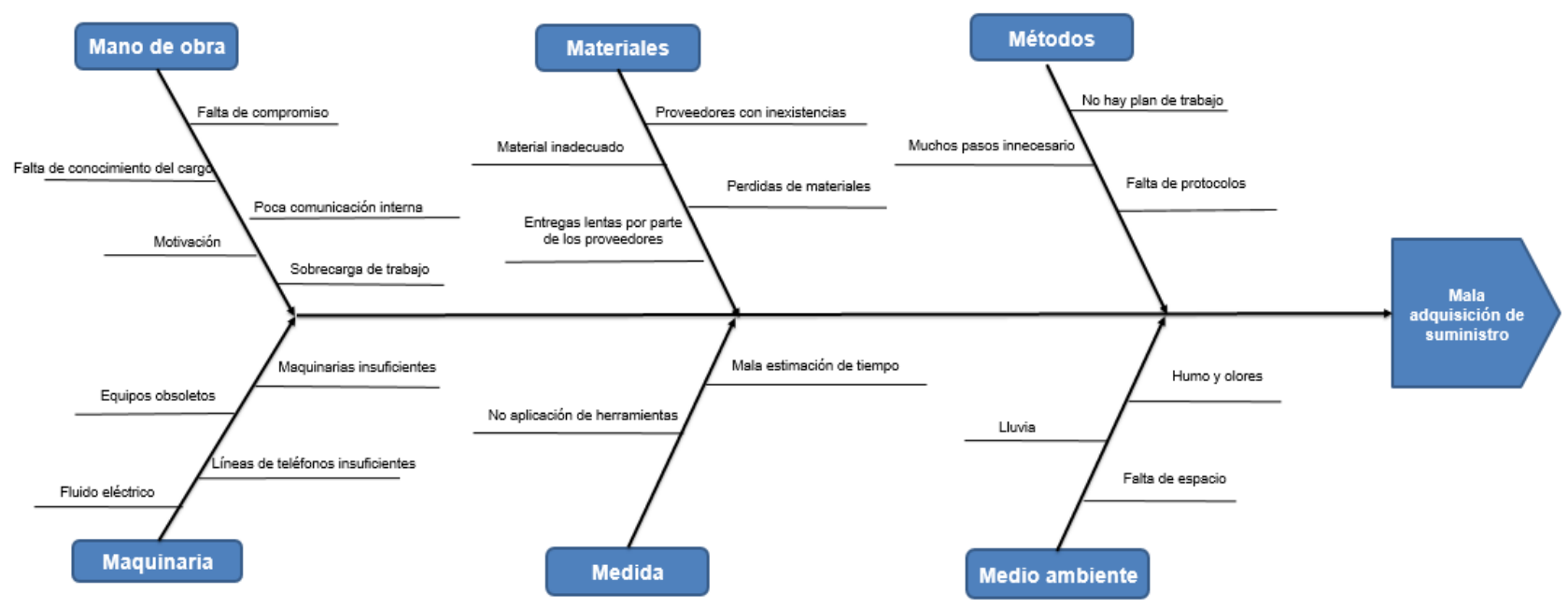

Figura 1. diagrama de Ishikawa de las dificultades en el aprovisionamiento de suministro en una empresa del sector eléctrico.

Tabla 1. Posibles causas que están afectando el aprovisionamiento de suministros.

\begin{tabular}{|l|c|}
\hline \multicolumn{1}{|c|}{ Causa / Problema / Fenómeno } & $\begin{array}{c}\text { Datos } \\
\text { recolectados }\end{array}$ \\
\hline Falta de conocimiento del personal & 20 \\
\hline Poca comunicación interna & 30 \\
\hline Equipos en mal estado & 25 \\
\hline Sobrecarga laboral & 30 \\
\hline Maquinaria insuficiente & 15 \\
\hline Inestabilidad en fluido eléctrico & 11 \\
\hline Mala estimación de tiempo & 25 \\
\hline Exceso de pasos en los procesos & 35 \\
\hline Plan de trabajo inexistente & 15 \\
\hline Espacio de trabajo inadecuado & 8 \\
\hline Falta de control en el sistema de inventarios & 20 \\
\hline Incumplimiento del horario del trabajo & 24 \\
\hline Intervención de los sindicatos & 10 \\
\hline Retrasos en pagos a proveedores & 19 \\
\hline
\end{tabular}

(C) The author; licensee Universidad de la Costa - CUC.

BILO vol. 3. no. 1 Enero - Junio, 2021

Barranquilla. ISSN Online 2711-3280. 


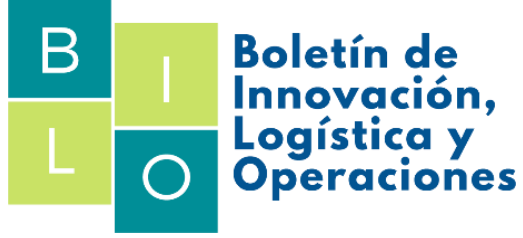

Posterior de la identificación de las causas se obtuvo la frecuencia con que ocurría estas, suministrado los siguientes resultados, presentado por medio de una tabla junto al diagrama de Pareto [9].

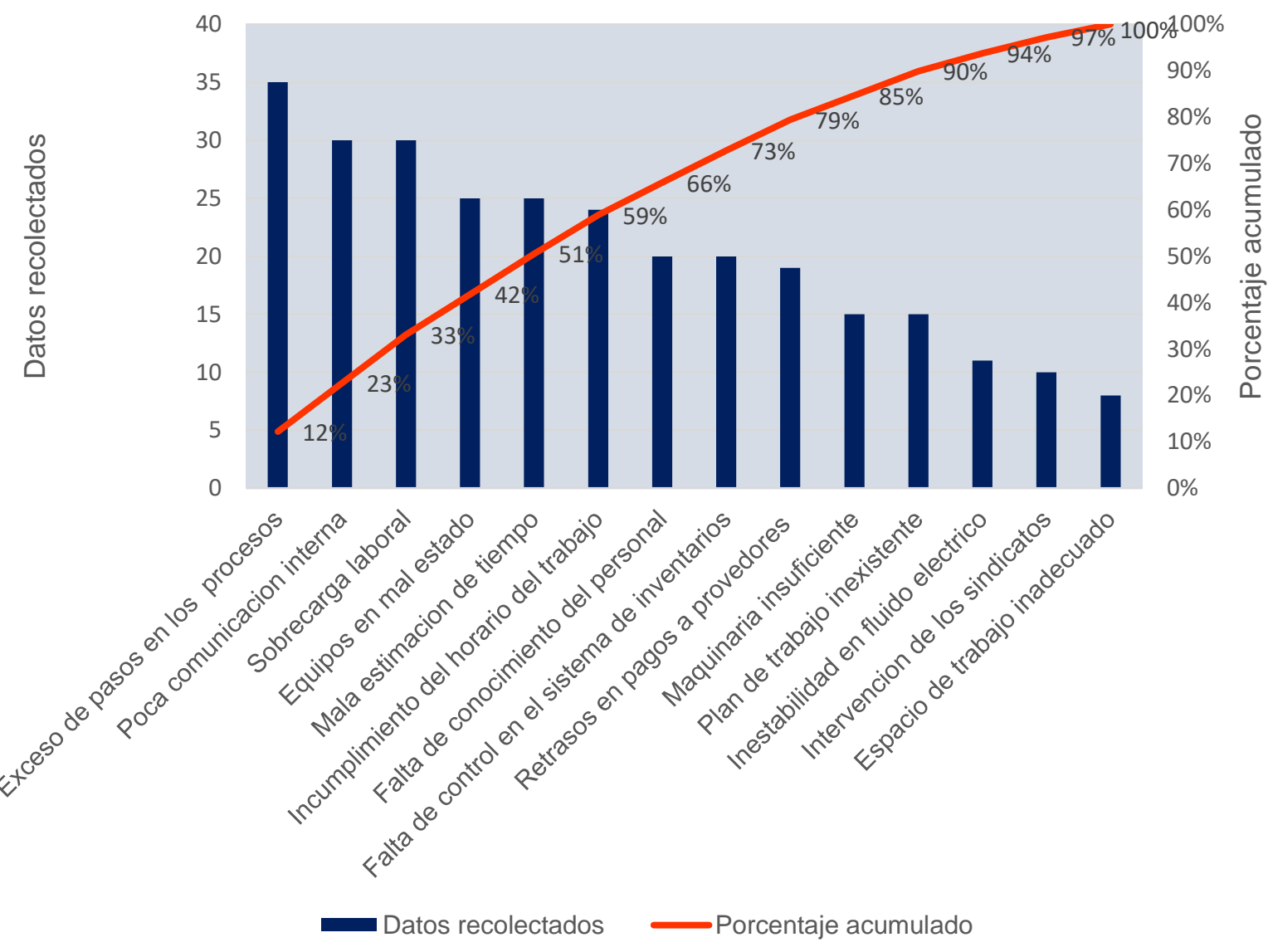

Gráfico 1. Identificación de las mayores causas mediante la implementación del diagrama de Pareto.

Con base del anterior diagrama de Pareto, se identificaron las causas potenciales que influyen en la dificultad en el aprovisionamiento de suministro. La utilización de esta herramienta permitió visualizar que el $80 \%$ de las fallas son ocasionadas principalmente por las causas exceso de pasos en los procesos y poca comunicación interna De esta manera se obtuvo cuáles deben ser las causas del problema que deben ser atacadas mediante un Plan de Mejora.

\section{Conclusión}

Se puede concluir que gracias a las herramientas de calidad implementadas (diagrama causa efecto de Ishikawa y Pareto) se identificaron satisfactoriamente las variables que afectaban la pérdida de tiempo en la adquisición de suministros de la empresa, hallando la causa del problema, el exceso de pasos en los procesos. La metodología recomendada es la siguiente, la persona encargada de obra

(C) The author; licensee Universidad de la Costa - CUC.

BILO vol. 3. no. 1 Enero - Junio, 2021

Barranquilla. ISSN Online 2711-3280. 
remita un correo con todos los suministros que requieran con antelación y la persona encargada del área de compras solicite a los proveedores y garantizar la llegada a tiempo a los respectivos frentes de trabajos, aplicando esta solución optimizamos los pasos para la adquisición de suministros. Se pudo observar la importancia que tiene estas herramientas de control de la calidad ya que facilitan en gran medida los procesos, garantizando la propuesta de las posibles soluciones para la problemática presentada.

\section{Referencias}

[1] F. Diaz, L. F. Gonzales Alon y F. Ximena, «Universidad señor de sipan,» 2020. [En línea]. Available: http://repositorio.uss.edu.pe/handle/uss/6992. [Último acceso: 1709 2020].

[2] P. A. Garzon Ulloa, D. V. Chicaiza Castillo, V. M. Pailiacho Mena y D. . J. Robayo Jácome, «Inteligencia de negocios en la gestión administrativa de una empresa distribuidora del sector eléctrico,» 3C TIC, 2020.

[3] G. Mordecki, «colibri,» 2020. [En línea]. Available: https://www.colibri.udelar.edu.uy/jspui/handle/20.500.12008/25031. [Último acceso: 17 septiembre 2020].

[4] M. R. Quevedo Barros, . L. . M. Vásquez Lafebre, J. . O. Quevedo Vázquez y L. . T. Pinzon Prado, «Dominio de las ciencias,» 2020.

[5] G. Wang, «Integrated supply chain scheduling of procurement, production, and distribution under spillover effects,» Computers and Operations Research, vol. 126, $\mathrm{n}^{\circ}$ 105105, Enero 2021.

[6] J. G. J. Izar, Las siete herramientas básicas de la calidad 1ed., San Luis Potosi, Mexico: Editorial Universitaria Potosina, 2004, p. 216.

[7] B. Salazar López, «Ingenieria Industrial,» 28 octubre 2019. [En línea]. Available: https://www.ingenieriaindustrialonline.com/gestion-de-calidad/las-siete-herramientas-de-la-calidad/. [Último acceso: 18 septiembre 2020].

[8] C. Ortiz Hernandez, A. Troncoso Palacio, D. Acosta Toscano, R. Begambre Meza y B. Troncoso Mendoza, «Utilización de herramientas de calidad para la mejora en los procesos de extrusión de plásticos,» 2019.

[9] M. Sales, 2019. [En línea]. Available: http://tecnicasdecep.pbworks.com/f/diagrama.de.Pareto.doc. [Último acceso: 18 septiembre 2020].

[10] K. Carrillo Herrera, «Estrategias sustentables en logística y cadenas de suministro,» 2017.

[11] L. A. Bearzotti, «Industria 4.0 y la gestión de la cadena de suministro: el desafio de la nueva revolucion industrial,» 2017.

[12] D. F. Batero Manso y J. A. A. Orjuela Castro, «El problema de ruteo e Inventarios en cadenas de suministro de perecederos: revisión de literatura,» 218.

[13] M. Pérez Gao Montoya, «Implementación de herramientas de control de calidad en,» 1101 2017. [En línea]. Available: https://www.redalyc.org/pdf/816/81653909013.pdf. [Último acceso: 1210 2020].

[14] J. Pereira, 19 diciembre 2018. [En línea]. Available: http://www.jpereira.net/gestion-de-colorarticulos/el-control-de-calidad-en-la-digitalizacio-n-de-bienes-culturales. [Último acceso: 19 septiembre 2020].

[15] G. Barone, noviembre 2017. [En línea]. Available: http://sedici.unlp.edu.ar/handle/10915/74064. [Último acceso: 15 octubre 2020].

(C) The author; licensee Universidad de la Costa - CUC.

BILO vol. 3. no. 1 Enero - Junio, 2021

Barranquilla. ISSN Online 2711-3280. 


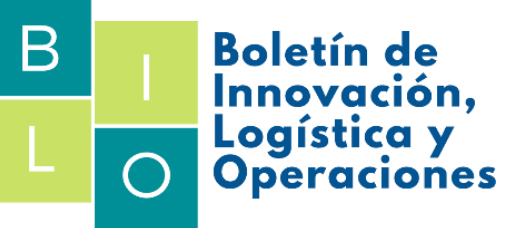

BILO Vol. 3 no. 1, Enero - Junio de 2021

[16] M. J. Cadme Chavez, «Diseño de un plan de control de calidad para la producción de yogur natural tipo I en una empresa ubicada en Durán.,» 4 marzo 2020. [En línea]. Available: http://192.188.52.94/handle/3317/14288. [Último acceso: 7 noviembre 2020].

[17] W. F. Peramas Abanto y E. A. Rios Oruna, «Mejoras en los procesos de la cadena de suministro para reducir tiempos de entrega en la exportación de Uva en la empresa Fegurri S.A.C,» 2020. [En línea]. Available: http://repositorio.upao.edu.pe/handle/upaorep/6556. [Último acceso: 7 octubre 2020].

[18] E. F. Novillo Maldonado y J. E. Feijoo Medina, «Herramienta de calidad diagrama causa y efecto aplicado al departamento de archivo general: caso Universidad Técnica de Machala.,» 2019.

[19] L. A. Castro Pereira y C. B. Sarmiento Chugcho, 05 febrero 2019. [En línea]. Available: http://repositorio.utmachala.edu.ec/handle/48000/13745.

[20] Senthilkumar y Nallusamy, «Enrichment of quality rate and output level in a medium scale manufacturing industry by implementation of appropriate quality tools,» 7 julio 2020 .

[21] K. Ishikawa, Introduccion al Control de Calidad, 1 ed., Madrid: Diaz de Santos, 1994, p. 500.

(C) The author; licensee Universidad de la Costa - CUC.

BILO vol. 3. no. 1 Enero - Junio, 2021

Barranquilla. ISSN Online 2711-3280. 